\title{
E.M. AND HADRONIC SHOWER SIMULATION WITH FLUKA
}

\author{
G. BATTISTONI ${ }^{1}$, A. FASSÓ ${ }^{2}$, A. FERRARI ${ }^{3,1}$, J. RANFT $^{4}$, A. RUBBIA ${ }^{5}$, \\ P.R. SALA ${ }^{5,1}$ \\ ${ }^{1}$ INFN, Sezione di Milano, via Celoria 16, 23100 Milano, Italy \\ E-mail: giuseppe.battistoni@mi.infn.it \\ ${ }^{2}$ SLAC, Stanford, CA 94309, USA \\ ${ }^{3}$ CERN, CH-1211 Geneva 23, Switzerland \\ ${ }^{4}$ Universität Siegen, 57068 Siegen, Germany \\ ${ }^{5}$ ETH, CH-8093 Zurich, Switzerland
}

A description of the main features of e.m. and hadronic shower simulation models used in the FLUKA code is summarized and some recent applications are discussed.

The general status of the FLUKA project is also reported.

\section{Introduction}

FLUKA ${ }^{1}$ is a multipurpose transport Monte Carlo code, able to treat hadron-hadron, hadron-nucleus, nucleus-nucleus, electromagnetic, $\mu$ and neutrino interactions up to $10000 \mathrm{TeV}$. Charged particle transport (handled in magnetic field too) includes all relevant processes. FLUKA is developed by implementing or including original and well tested microscopic models and its performances are optimized comparing to particle production data at single interaction level. No tuning whatsoever is performed on "integral" data, such as calorimeter resolutions, thick target yields, etc. Results in complex cases, as well as scaling laws and properties, come forth naturally from the underlying physical models and the basic conservation laws are fulfilled a priori. Whenever possible analytical sampling is preferred to rejection techniques, in order to optimize speed, while, in order to maintain a high degree of accuracy, lookup tables have been privileged with respect to analytical approximation. Energy is conserved in each process within the computer accuracy. Here we review, also by means of application examples, some of the FLUKA features which are interesting for calorimetry. 


\section{Models for E.M. and Hadronic Shower Simulation}

For historical reasons, FLUKA is best known for its hadron event generators, but since more than 10 years FLUKA can handle with similar or better accuracy electromagnetic effects. Nowadays, as far as e.m. interactions are concerned, different programs are often found to be in similar good agreement with experimental data in most typical routine cases. However, FLUKA still offers an approach which makes it an interesting alternative for a number of specialized problems. A general review of the e.m. part of FLUKA can be found elsewhere ${ }^{2}$. Briefly, the energy range covered by this sector of FLUKA is very wide: the program can transport photons and electrons over about 12 energy decades, between $1 \mathrm{PeV}$ and $1 \mathrm{keV}$. The e.m. part is fully coupled with the hadron sector, including the low (i.e. < $20 \mathrm{MeV}$ ) neutrons. In particular, photonuclear reactions are implemented from threshold up to the maximum energy of the FLUKA hadron event generator (about $20 \mathrm{TeV}$ ). Attention has been given to an accurate description of the most important electron and photon interactions, reproducing correlations as far as possible and avoiding unnecessary approximations. A special care has been put in the description of both very high and very low energy effects (LPM effect, Ter-Mikaelyan effect, single scattering transport, photoelectron angular distribution, fluorescence).

A recent review of hadronic interaction models in FLUKA can be found elsewhere $^{3}$. Here we limit ourselves to remind that basically one starts from hadron-nucleon interactions, always according to the general "microscopic" approach, to arrive eventually to hadron-nucleus and nucleus-nucleus collision models. Elastic, charge exchange and strangeness exchange reactions are described as far as possible by phase-shift analysis and/or fits of experimental differential data. As soon as inelastic hadron-nucleon interactions are concerned, two families of models are adopted, depending on the projectile energy, the former is based on individual resonance production and decays ${ }^{4}$, and covers the energy range up to $3-5 \mathrm{GeV}$, while the latter, for higher energy, is based on the Dual Parton Model ${ }^{5}$ (DPM), a particular quark/parton string model, and provides reliable results up to several tens of $\mathrm{TeV}$. Further details can be found elsewhere ${ }^{6}$. At energies high enough to consider coherent effects as corrections, a hadron-nucleus reaction can be described as a cascade of two-body interactions, concerning the projectile and the reaction products. This is the mechanism called IntraNuclearCascade (INC), which in FLUKA also incorporates many quantum effects (see

$\left.{ }^{1,6}\right)$. All these items are accurately implemented in the low-intermediate 
energy model of FLUKA, called PEANUT (PreEquilibrium-Approach-toNUclear-Thermalization). The INC goes on in PEANUT until all nucleons are below a smooth threshold around $50 \mathrm{MeV}$, and all particles but nucleons (typically pions) have been emitted or absorbed. The nuclear configuration at this point is used as starting condition for the preequilibrium stage ${ }^{6}$. For projectile energies higher than $3-5 \mathrm{GeV}$, the first inelastic interaction is described according to the Glauber-Gribov ${ }^{7,8}$ model. The nuclear effects on the secondaries are taken into account by a simplified, generalized INC. At the end of the reaction chain, the nucleus is treated as a thermally equilibrated system, characterized by its excitation energy. Evaporation and/or fission follows ${ }^{9}$.

Recently FLUKA has been successfully extended to nucleus-nucleus collisions, using the DPMJET-II. $53^{10}$ code at high $(>5 \mathrm{GeV} / \mathrm{n})$ energies. DPMJET-II.53 is a Monte Carlo model based, as FLUKA, on DPM and Glauber formalism, for sampling hadron-hadron, hadron-nucleus and nucleus-nucleus collisions for $\mathrm{E}_{l a b}$ up to $10^{9}-10^{11} \mathrm{GeV} / \mathrm{n}$ ). In the lower energy range, FLUKA has been interfaced to an extensively modified version of the relativistic Quantum-Molecular-Dynamics model RQMD-2.4 code $^{11}$. Examples of the performance of FLUKA connected to RQMD-2.4 have been presented in ${ }^{12}$

\section{Application of FLUKA to E.M. Shower Simulation}

Among the most recent applications of FLUKA in e.m. shower simulation, the case of the ICARUS experiment ${ }^{13}$ has some relevance. ICARUS is based on the concept of the high purity Liquid Argon TPC, with electron drifting over $1.5 \div 2 \mathrm{~m}$. It is designed to perform precise measurements on rare event physics (nucleon decay, neutrino interactions, etc). The sensitive Liquid Argon volume can be thought as a homogeneous, low density ( $\rho$ $=1.4 \mathrm{~g} / \mathrm{cm}^{3}$ ) calorimeter having $X_{0}=14 \mathrm{~cm}$. In ICARUS FLUKA is being extensively used for detector simulation: a precision detector requires high quality detector simulation. However, beyond the accuracy of physics implementation, the accurate description of specific detector effects has a relevant impact on the quality of results. In this specific case: i) the charge attenuation as a function of drift distance and ii) the charge recombination probability ("quenching") as a function of ionization density and electric field. The second of these two aspects required a detailed work in order to find the correct quenching parameters ${ }^{14}$. These cannot be put in the form of a global correction to be applied to a whole track by means of a unique Birks' law-like correction, but instead they must be applied to each energy 
deposition step. The step by step correction parameters are found to be a function of the $\delta$-ray production threshold. Figure 1 shows the capability of reproducing the overall quenching factor as a function of $d E / d x$ value for electrons on a sample of data from the 3 ton test module of ICARUS, for an electric field value of $350 \mathrm{~V} / \mathrm{cm}(\delta$-ray cutoff at $10 \mathrm{keV})$. The coefficient were obtained from the MC comparison to the data taken in the T600 test, at $500 \mathrm{~V} / \mathrm{cm}$. One of the most important output of simulation studies

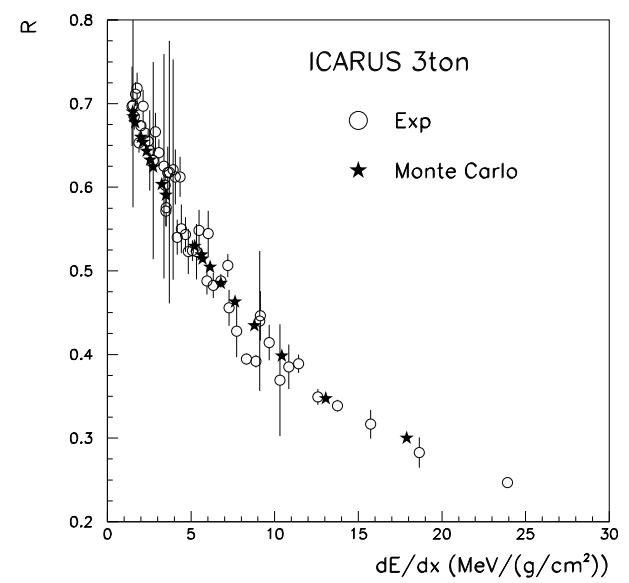

Figure 1. Experimental and Monte Carlo recombination factors in ICARUS.

with FLUKA in ICARUS concerns the evaluation of the $\mathrm{e} / \pi_{0}$ separation capability in order to learn how to distinguish $\nu_{e} n \rightarrow e p$ from $\nu_{x} n \rightarrow \nu$ $\pi_{0} X$. This is a crucial feature for present neutrino oscillation studies, and the previous work on quenching corrections was an essential step in this. The measurement of $d E / d x$ in the first millimeters of the showering events allows to distinguish between one and two m.i.p. levels in the first wires (Figure 2). If the vertex of the event is known, knowing the mean free path of a photon for pair production $\left(9 / 7 X_{0}\right)$ in liquid argon is $\sim 18 \mathrm{~cm}$, a pion can be distinguished if it converts beyond $1 \mathrm{~cm}$. At $1 \mathrm{GeV}$ only $0.2 \%$ of $\pi_{0}$ contamination, for an electron efficiency of $90 \%$, is thus predicted.

\section{Application of FLUKA to hadronic shower simulation}

Beyond the ICARUS detector simulation, one of the most recent interesting applications of FLUKA concern cosmic ray physics, and in particular 


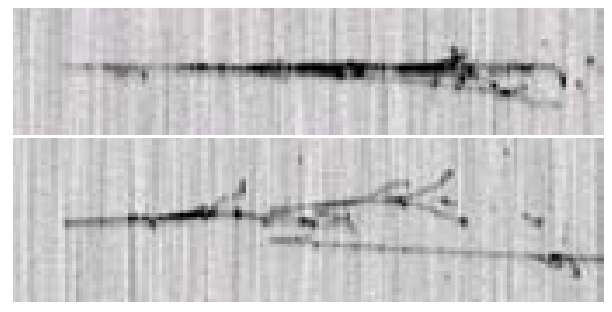

Figure 2. FLUKA simulated imaging of and electron (top) and a pion (bottom) event at $1 \mathrm{GeV}$ in the T600 ICARUS module. The grey scale represents the deposited energy. Each pixel represents a wire hit $(3 \mathrm{~mm}$ pitch). The single mip track of electron and the double mip track of $e^{-} e^{+}$from $\pi^{0}$ decay are visible in the first wires.

the study of hadronic showers atmosphere, a homogeneous, but with variable density, calorimeter having a depth of about $10 \lambda$. For this purposes a dedicated FLUKA library has been developed, together with additional packages including a set of primary cosmic ray spectra from $\mathrm{Z}=1$ to $\mathrm{Z}=28$ (derived from NASA and updated to most recent measurements), a solar modulation model correlated to neutron monitors counts, an atmospheric model, a 3D geometry of earth+atmosphere, a geomagnetic model ${ }^{15}$. Secondary charged/neutral particles can be recorded in atmosphere at different altitudes. This package is being benchmarked with data on muon and hadron fluxes (at ground level and various altitudes) and is used for a research projects concerning atmospheric neutrinos ${ }^{16}$ and dosimetry in civil aviation ${ }^{17}$. A collaboration has been started with Karlsruhe so that the hadronic sector (only $E>50 \mathrm{MeV}$ ) of FLUKA is now a possible option of the CORSIKA package ${ }^{18}$ for $\mathrm{E}_{\text {had }}<80 \mathrm{GeV}^{19}$.

\section{Conclusions: status of FLUKA project and future developments}

FLUKA, which is being developed within INFN since 1989, became an official INFN project in 2002, carried on in strict collaboration with CERN and The University of Houston. Further information can be found in the FLUKA web site (http://www.fluka.org). In 2003 FLUKA has become a joint INFN-CERN project to share the responsibility of developing, maintaining and distributing the FLUKA code. Technical and physics developments are in preparation: suppression of the separate E.M. preprocessor, photo-production of $\mu$ pairs, "Doppler broadening" of Compton scattering, LPM effect in pair-production, Monte Carlo realization of the formalism of Boltzman Master Equation ${ }^{20}$ for A-A scattering for $\mathrm{E}<100 \mathrm{MeV} / \mathrm{A}$, inter- 
face to DPMJET III ${ }^{21}$, introduction of a more self-consistent QMD model. A deep revision of the packages for Cherenkov, scintillation and transition radiation light production and transport in FLUKA, also useful for calorimeter simulation, is also in progress. One of us (A. Fassò) acknowledges the partial support by the US D.o.E. contract DE-AC03-76SF00515.

\section{References}

1. A. Fassò et al., in Proc. of SARE-3, KEK-Tsukuba, May 7-9 1997, KEK report Proc. 97-5, 32 (1997); A. Fassò et al., Proc. of the Monte Carlo 2000 Conf., Lisbon, October 2000, Springer-Verlag Berlin, 955 (2001).

2. A. Fassò, et al., Proc. of the Monte Carlo 2000 Conf., Lisbon, October 2000, Springer-Verlag Berlin, 159 (2001).

3. A. Fassó, et al., "The physics models of FLUKA: status and recent developments", 2003 Conf. of Computing in High-Energy and Nuclear Physics (CHEP 03), La Jolla, California, 24-28 Mar 2003. e-Print Archive: hep-ph/0306267

4. K. Hänssgen, and J. Ranft, Nucl. Sci. Eng. 88, 537 (1984); S. Huber, and J. Aichelin, Nucl. Phys. A573, 587 (1994); A. Engel, W. Cassing, U. Mosel, M. Schäfer, Gy. Wolf, Nucl. Phys. A572, 657 (1994).

5. A. Capella, et al., Phys. Rep. 236, 225 (1994).

6. A. Ferrari, and P.R. Sala, Proc. of Workshop on Nuclear Reaction Data and Nuclear Reactors Physics, Design and Safety, Trieste, Italy, April 1996, 2, 424 (1998).

7. R.J. Glauber and G. Matthiae, Nucl. Phys. B21, 135 (1970).

8. V.N. Gribov, Sov. Phys. JETP 29, 483 (1969).

9. A. Ferrari, P.R. Sala, J. Ranft, and S. Roesler, Z. Phys. C70, 413 (1996).

10. J. Ranft, Phys. Rev. D51, 64 (1995).

11. H. Sorge, Phys. Rev. C52, 3291 (1995).

12. F. Ballarini et al., Proc. of the $10^{t h}$ Int.l Conf. on Nuclear Reaction Mechanisms, June 2003, Varenna, Italy, E. Gadioli ed., Univ. Studi di Milano, 579 (2003)

13. F. Arneodo et al. (ICARUS Collaboration), A second-Generation Proton Decay Experiment and Neutrino Observatory at the Gran Sasso Laboratory, LNGS-P28/2001

14. S. Amoruso et al. (ICARUS Collaboration), Nucl Inst. \& Meth. A 523, 275 (2004)

15. http://nssdc.gsfc.nasa.gov/space/model/models/igrf.html

16. G. Battistoni et al, Astropart.Phys. 19 (2003) 269, Erratum-ibid.19:291.

17. M. Pelliccioni, Radiat. Protec. Dosim. 88, 279, 2000.

18. D. Heck, et al., Report FZKA 6019 (Forshungszentrum Karlsruhe), http://www-ik.fzk.de/ heck/corsika

19. D. Heck et al., Proc. 28th ICRC, Tsukuba, Japan, 2003, HE-1.2, 279 (2003).

20. M. Cavinato, et al., Nucl. Phys. A679, 753 (2001).

21. S. Roesler, R. Engel and J. Ranft, Proc. of Monte Carlo 2000 Conf., Lisbon, October 2000, Springer-Verlag Berlin, 1033 (2001). 